\title{
CANONICAL STRUCTURES FOR PALINDROMIC MATRIX POLYNOMIALS
}

\author{
Peter Lancaster, Uwe Prells And Leiba Rodman
}

\begin{abstract}
Spectral properties and canonical structures of palindromic matrix polynomials are studied in terms of their linearizations, standard triples, and unitary triples. These triples describe matrix polynomials via eigenvalues and Jordan chains. As an application of canonical structures and their properties, criteria are developed for stable boundedness of solutions of systems of linear differential equations with symmetries.
\end{abstract}

Mathematics subject classification (2000): 47A56, 15A22, 39A99. equations.

Key words and phrases: Matrix polynomials, palindromic, linearizations, spectral triples, difference

\section{REFERENCES}

[1] ANTONIOU, E. N., AND VologianNidis, S., A new family of companion forms of polynomial matrices, Elec. J. of Linear Algebra, 11 (2004), 78-87.

[2] FIEDLER, M., A note on companion matrices, Linear Algebra and its Applications, 372 (2003), 325-331.

[3] GOHBERG, I., KAASHOEK, M. A., AND LANCASTER, P., General theory of regular matrix polynomials and band Toeplitz operators, Integral Eq. and Op. Theory, 11 (1988), 776-882.

[4] Gohberg, I., Lancaster, P. and Rodman, L., Perturbations of $H$-selfadjoint matrices, with applications to differential equations, Integral Eq. and Op. Theory 5 (1982), 718-757.

[5] Gohberg, I., LANCASter, P. And Rodman, L., Matrices and Indefinite Scalar Products, Birkhäuser, Basel, 1983.

[6] Gohberg, I., Lancaster, P. And Rodman, L., Indefinite Linear Algebra and its Applications, Birkhäuser, Basel, 2005.

[7] Gohberg, I., LANCASTER, P. AND Rodman, L., Spectral analysis of selfadjoint matrix polynomials, Annals of Mathematics, 112 (1980), 33-71.

[8] Gohberg, I., Lancaster, P. And Rodman, L., Matrix Polynomials, Academic Press, 1981.

[9] Gohberg, I., AND SEmencul, A. A., On inversion of finite-section Toeplitz matrices and their continuous analogues, (Russian), Mat. Issled., Kishinev, 7 (1972), 201-224.

[10] HeInIG, G., AND Rost, K., Algebraic Methods for Toeplitz-like Matrices and Operators, AkademieVerlag, Berlin, 1984.

[11] KaIlath, T., AND Koltracht, I., Matrices with block-Toeplitz inverses, Linear Algebra and its Applications, 75 (1986), 145-153.

[12] LANCASTER, P., AND PRELLS, U., Isospectral vibrating systems. II. Structure preserving transformations, Oper. Theory Adv. Appl., 163 (2006), 275-298, Birkhäuser, Basel.

[13] LANCASTER, P., AND PRELLS, U., Isospectral families of high-order systems, Zeit. für Ang. Mathematik und Mechanik, 87 (2007), 219-234.

[14] Lander, F. I., The Bezoutiant and the inversion of Hankel and Toeplitz matrices, (Russian) Mat. Issled., 9 (1974), 69-87; 249-250.

[15] Mackey, D. S., Mackey, N., Mehl, C., And Mehrmann, V., Structured polynomial eigenvalue problems: Good vibrations from good linearizations, SIAM J. Matrix Anal. and Appl., 28 (2006), $1029-1051$. 
[16] Mackey, D. S., Mackey, N., Mehl, C., And Mehrmann, V., Vector spaces of linearizations for matrix polynomials, SIAM J. Matrix Anal. and Appl., 24 (2006), 971-1004.

[17] RoDman, L., Bounded and stably bounded palindromic difference equations of first order, Elec. J. of Linear Algebra, 15 (2006), 22-49. 